\title{
Intermediate-mass black holes in globular clusters: observations and simulations - Update
}

\author{
Nora Lützgendorf ${ }^{1}$, Markus Kissler-Patig ${ }^{2}$, Karl Gebhardt ${ }^{3}$, Holger \\ Baumgardt $^{4}$, Diederik Kruijssen ${ }^{5}$, Eva Noyola ${ }^{3}$, Nadine Neumayer ${ }^{6}$, \\ Tim de Zeeuw ${ }^{7,8}$, Anja Feldmeier-Krause ${ }^{7}$, Edwin van der Helm ${ }^{8}$, Inti \\ Pelupessy $^{8}$ and Simon Portegies Zwart ${ }^{8}$ \\ ${ }^{1}$ ESA, Space Science Department, Keplerlaan 1, \\ NL-2200 AG Noordwijk, The Netherlands \\ email: nluetzge@cosmos.esa.int \\ ${ }^{2}$ Gemini Observatory, Northern Operations Center, 670 N. A'ohoku Place, \\ Hilo, Hawaii, 96720, USA \\ ${ }^{3}$ Department of Astronomy, University of Texas at Austin, Austin, \\ TX 78712, USA \\ ${ }^{4}$ School of Mathematics and Physics, University of Queensland, \\ Brisbane, QLD 4072, Australia \\ ${ }^{5}$ Max-Planck Institut für Astrophysik, Karl-Schwarzschild-Straße 1, \\ D-85748, Garching, Germany \\ ${ }^{6}$ Max-Planck-Institute for Astronomy, Königstuhl 17, \\ 69117, Heidelberg, Germany \\ ${ }^{7}$ European Southern Observatory, Karl-Schwarzschild-Straße 2, \\ D-85748 Garching, Germany \\ ${ }^{8}$ Leiden Observatory, Leiden University, PO Box 9513, \\ NL-2300 RA, Leiden, The Netherlands
}

\begin{abstract}
The study of intermediate-mass black holes (IMBHs) is a young and promising field of research. If IMBH exist, they could explain the rapid growth of supermassive black holes by acting as seeds in the early stage of galaxy formation. Formed by runaway collisions of massive stars in young and dense stellar clusters, intermediate-mass black holes could still be present in the centers of globular clusters, today. We measured the inner kinematic profiles with integral-field spectroscopy for 10 Galactic globular cluster and determined masses or upper limits of central black holes. In combination with literature data we further studied the positions of our results on known black-hole scaling relations (such as $M_{\bullet}-\sigma$ ) and found a similar but flatter correlation for IMBHs. Applying cluster evolution codes, the change in the slope could be explained with the stellar mass loss occurring in clusters in a tidal field over its life time. Furthermore, we present results from several numerical simulations on the topic of IMBHs and integral field units (IFUs). N-body simulations were used to simulate IFU data cubes. For the specific case of NGC 6388 we simulated two different IFU techniques and found that velocity dispersion measurements from individual velocities are strongly biased towards lower values due to blends of neighbouring stars and background light. In addition, we use the Astrophysical Multipurpose Software Environment (AMUSE) to combine gravitational physics, stellar evolution and hydrodynamics to simulate the accretion of stellar winds onto a black hole. We find that the S-stars need to provide very strong winds in order to explain the accretion rate in the galactic center.
\end{abstract}

Keywords. stars: kinematics and dynamics, methods: numerical, black hole physics 


\section{Introduction}

Intermediate-mass black holes (IMBHs) provide the missing link between supermassive black holes and stellar-mass black holes. Their masses range from a few hundred solar masses up to $10^{5} M_{\odot}$. Possible formation scenarios are remnants of population III stars (Madau \& Rees 2001) or runaway merging in young dense star clusters (e.g. Portegies Zwart et al. 2004). Detecting and measuring these black holes would provide important data points to the $M_{\bullet}-\sigma$ relation at the lower mass end. Furthermore, IMBHs could explain the rapid growth of supermassive black holes at high redshift by acting as seeds in the early universe.

Many attempts have been made to detect IMBHs in globular clusters. These range from spectroscopic and photometric velocity measurements (e.g. Noyola et al. 2008) to X-ray and radio observations in order to find signatures of accretion (e.g. Strader et al. 2012). The results are disputed and the question whether IMBHs exist in globular clusters is not yet resolved. Each method has its caveats. The low gas content in globular clusters makes the detection of accretion signatures rather difficult. The X-ray and radio signals from low and irregular accretion rates are not yet well understood. On the other hand, kinematic signatures suffer from shot noise caused by a few bright stars or contamination from background light. It is therefore crucial to advance the field of IMBHs in all directions. Simulations on globular clusters and observing techniques provide a valuable tool to verify observing results and to understand the internal processes of globular clusters.

Previous numerical work on IMBHs in globular clusters has been performed by Baumgardt et al. (2005) and Noyola \& Baumgardt (2011) who found that the surface brightness profiles of clusters hosting an IMBH exhibit weak central cusps, in contrast to corecollapsed clusters with very steep profiles and pre-core collapsed systems with no cusp at all. However, Trenti et al. (2010) and Vesperini \& Trenti (2010) showed that weak cusp profiles could be confused with that of clusters that are on their way to core-collapse, hence the density profile alone is not a sufficient criterion for a globular clusters hosting an IMBH. Furthermore, it has been shown that IMBHs prevent a cluster from undergoing core collapse and reduce the degree of mass segregation compared with non-IMBH clusters (Baumgardt et al. 2004; Gill et al. 2008). Trenti et al. (2007) predicted that clusters with a high ratio of core radius to half-mass radius are good candidates for hosting an IMBH. This was challenged by Hurley (2007), who showed that the ratios observed for Galactic globular clusters can be explained without the need for an IMBH when treating model data as if they were observational data.

\section{Observations}

We used integral field spectroscopy provided by the FLAMES (Fiber Large Array Multi Element Spectrograph, Pasquini et al. 2002) instrument mounted on UT2 at the Very Large Telescope (VLT) to obtain integrated light spectra from 10 Galactic globular clusters. In addition to the spectroscopic data we used HST images from the archive for each cluster. From these images we obtained the color magnitude diagram, the photometric center and the surface brightness profile.

The velocity dispersion profile was then used to determine the possible existence and mass of an intermediate-mass black hole in the center. For this, the derived density profile of each cluster was used as an input for Jeans models. By applying $\chi^{2}$ statistics we found the model that fits the data best. Figure 1 shows the fit of the globular cluster NGC 6266 with an IMBH signature. The profile clearly rises and requires a model with a black-hole mass of $\sim 3000 M_{\odot}$. This method was applied to all clusters in the sample and for each 


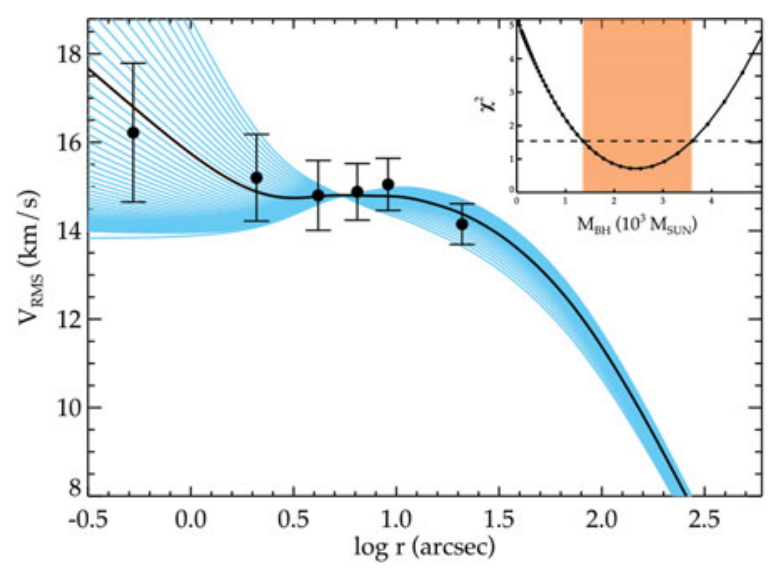

Figure 1. Velocity-dispersion profile of NGC 6266 overplotted by Jeans models with different black-hole masses. The $\chi^{2}$ values is shown in the upper right and the best fit model indicated by the black sold line.

one we reported an upper limit or a value on the black hole mass (Lützgendorf et al. 2011, 2012, 2013b; Feldmeier et al. 2013).

Using the data points and upper limits of our sample together with measurements from the literature, we compared the correlation between black-hole mass and host system properties such as velocity dispersion and total mass with those measured for supermassive black holes in galaxies. In Lützgendorf et al. (2013c) we analyzed this small and challenging data set by applying survival analysis in combination with Markov-chain Monte Carlo in order to account for uncertainties and upper limits. We found that the major correlations are prominent but shallower than those for supermassive black holes (Figure 2). In a follow up paper (Kruijssen \& Lützgendorf 2013) we showed that a possible explanation for the offset and more shallow correlation is the severe mass loss and expansion of globular clusters during their life time in a tidal field which leads to a reduction of the velocity dispersion and total mass of the system (see Figure 2).

\section{Simulations}

Because of large uncertainties in the measurements and contradicting results from different methods in the field of IMBHs, it is crucial to support the observing strategies, analysis and physics of the observations with sophisticated simulations. The next sections describe the different simulations that were performed to compare observations and verify our observing techniques.

\subsection{IFU simulations}

Integral field spectroscopy has become a popular tool in astronomy over the last years. The ability of simultaneously retrieving spatial and spectral data of an object has significant advantages for dynamical and stellar population studies. However, it is not yet clear what is the effect of this observing method on semi-resolved systems such as galactic globular clusters. For this reason we have developed a tool that allows to simulate IFU observations using star catalogues for different distances and observing conditions (i.e. seeing, Strehl ratio). The program uses a Moffat PSF that is applied to each star and integrated in the grid of the IFU. Using N-body simulations and N-body realizations we have tested the outcome of IFU observations for clusters with different properties, distances, and seeing. The results will be presented in Lützgendorf et al. (in prep). 

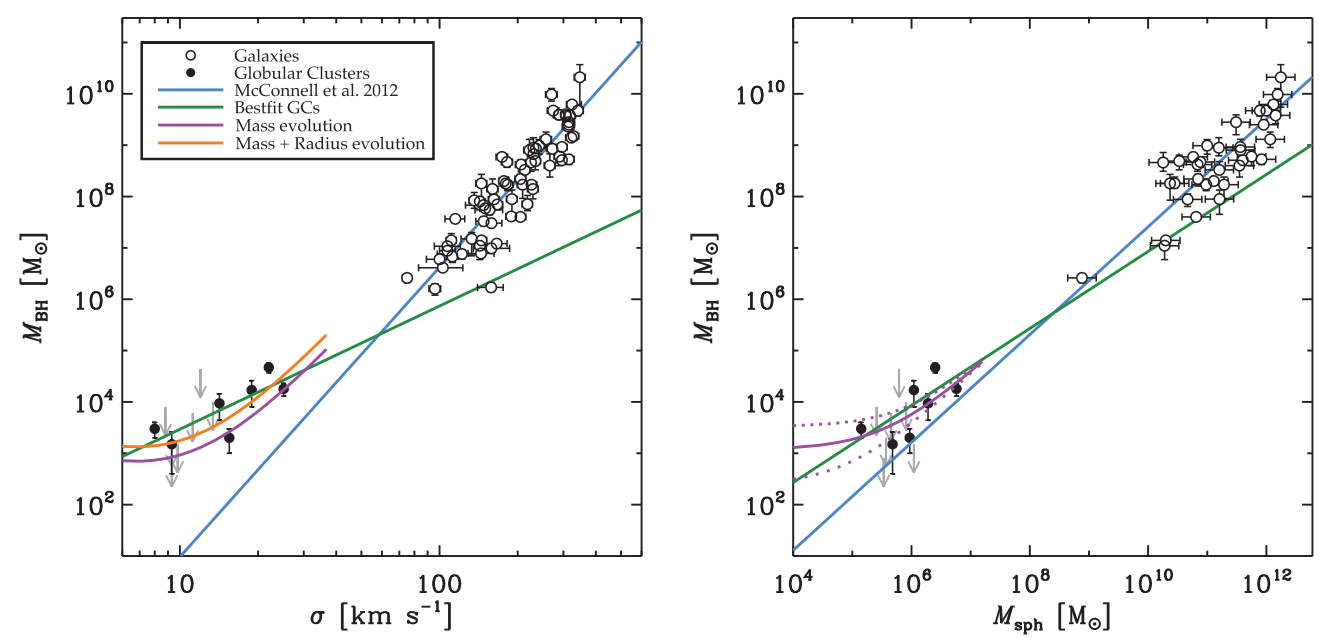

Figure 2. $M_{\bullet}-\sigma$ and $M_{\bullet}-M$ relation for supermassive black holes and IMBHs. The clear offset and shallower slope is well reproduced by cluster evolution models considering mass evolution only (magenta line) as well as models that additionally take the radius evolution (i.e. expansion) into account (orange line Kruijssen \& Lützgendorf 2013).
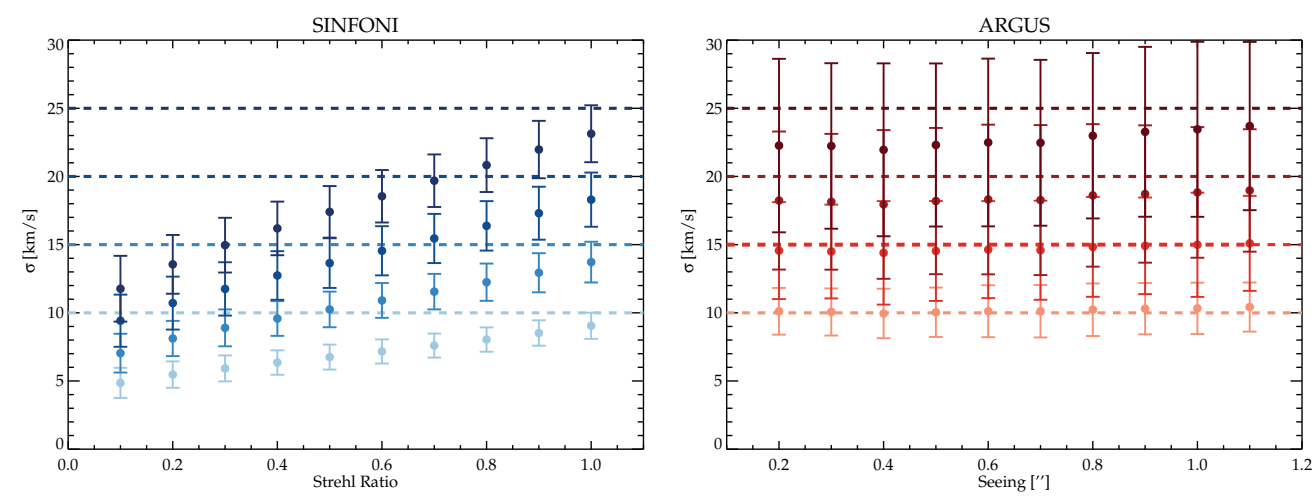

Figure 3. IFU simulations for SINFONI and ARGUS (central bin) with different input velocity dispersions as a function of Strehl ratio (the amount of light contained in the diffraction-limited core of the PSF, with respect to the total flux) and seeing. The input velocity dispersions are shown as dashed lines, the measured velocity dispersions as dots in the corresponding color.

In addition we investigated the specific case of NGC 6388 where two different methods using IFUs brought very different results. While Lützgendorf et al. (2011) found a steeply rising velocity dispersion profile and therefore a strong signature for an IMBH in its center, Lanzoni et al. (2013) measured a central velocity dispersion by taking individual velocities from adaptive optics supported SINFONI observations that is $40 \%$ lower than the value measured with integrated light. We reproduced both observations using the IFU simulation code and find the SINFONI observations being biased towards lower velocity dispersions due to blends of neighboring stars and background light (Lützgendorf et al. 2015, see Figure 3).

\subsection{AMUSE simulations}

Recently, many IMBH detections were challenged by contradicting measurements from different observing techniques. Especially the discrepancy between kinematic black-hole measurements and the absence of strong X-ray and radio emission from the centers of 

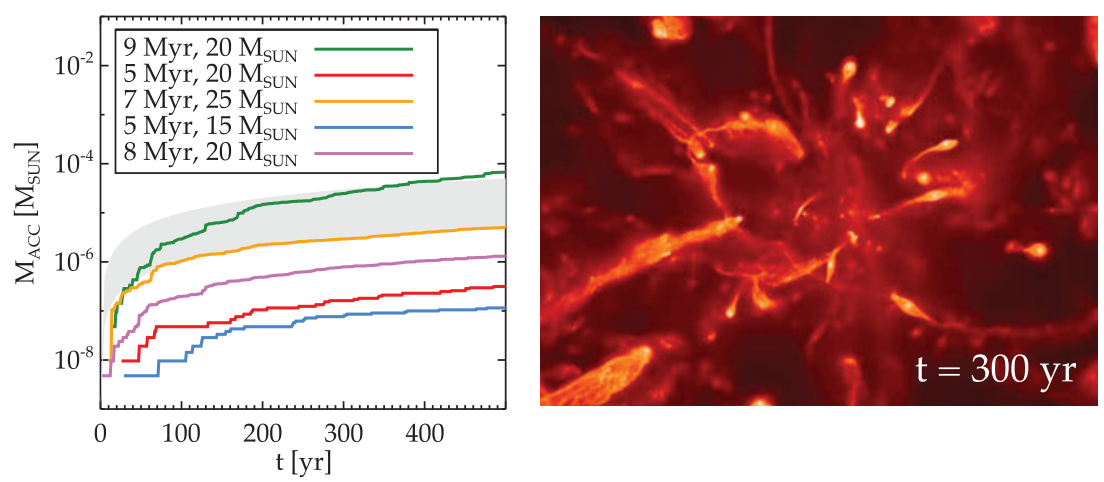

Figure 4. Left: Black-hole accretion rates for different stellar parameters. The gray shaded area marks the observed range. Right: Snapshot of the AMUSE simulation showing all 27 S-Stars and their stellar winds while orbiting the supermassive black hole in the center.

globular clusters remain an unsolved mystery (Strader et al. 2012). The main uncertainty when translating X-ray and radio flux measurements to black-hole masses is the amount of gas that is accessible for the black hole to accrete. We set out to investigate the effect of stellar winds on the accretion flow of the black hole. By using the Astrophysical Multipurpose Software Environment (AMUSE, Portegies Zwart et al. 2009, 2013; Pelupessy et al. 2013) we combined gravitational physics, stellar evolution and hydrodynamics into a single simulation of stars interacting with a black hole in the center of a globular cluster. The first application of this code will be presented in Lützgendorf (submitted to MNRAS) where the accretion rate of the supermassive black hole in the Milky Way from stellar winds of the surrounding S-Stars is studied (Figure 4, right panel).

We find that a) the total accretion is lower when summing up the contributions of individual stars (i.e. when running the simulation for each star separately, measuring the accretion and summing them up) than when including all stars in the simulation, b) cooling is inefficient and the temperatures are high due to wind shocks, and c) the accretion rate is highly dependent on the stellar parameters. In Figure 4 we show the accretion rates for different stellar parameters. The gray area marks the observed accretion rate from various sources in the literature (e.g. Baganoff et al. 2003; Bower et al. 2003; Nayakshin 2005). In order to reproduce the observed accretion rate of $10^{-8}-10^{-7} M_{\odot}$ year $^{-1}$ the stars have to be in their late evolutionary phase with massive winds, masses of $M=19-25 M_{\odot}$ and ages between 7 and 10 Myr. This, however, is in contrast with observations that have shown that these stars are rather young, main-sequence B-stars. We therefore conclude that the S-stars cannot account for the accretion rate alone.

\section{Summary}

We present the work done by our group on the field of IMBHs in globular clusters over the past years. Using integral field spectroscopy in combination of high-resolution HST imaging we have investigated the presence of IMBHs in a sample of 10 galactic globular clusters. We found about a third of them show signatures of an IMBH in the center. Using our data points in combination with literature data we put the IMBH mass estimates in context with properties of their host systems and compared this to existing scaling relations. We found an offset, shallower correlation for the main relations $\left(M_{\bullet}-\sigma, M_{\bullet}-L\right.$ and $\left.M_{\bullet}-M\right)$ which can be explained by the mass loss of globular clusters located in a tidal field over its life time. To support our observations we performed N-body simulations 
on globular clusters with and without a central IMBH. Further simulations aimed on reproducing IFU simulations by creating fake data sets from realistic N-body simulations and realizations with different distances and observing conditions. The simulations show that internal properties are in general well reproduced with IFU observations but that large uncertainties due to shot noise are unavoidable. In the specific case of NGC 6388 we found a strong bias in the velocity dispersion when computing it from individual velocities due to blend effects and background contamination. Finally, we used the Astrophysical Multipurpose Software Environment (AMUSE) to simulate the accretion of stellar winds onto a supermassive black hole to reproduce the S-Star system in the galactic center. We find that in order to explain the accretion rate the stars have to exhibit very massive stellar winds which collides with observational data. We plan to apply the code developed here to globular clusters and intermediate mass black holes in future work.

\section{References}

Aarseth, S. J. 1999, PASP, 111, 1333

Baganoff, F. K., Maeda, Y., Morris, M., et al. 2003, ApJ, 591, 891

Baumgardt, H., Makino, J., \& Ebisuzaki, T. 2004, ApJ, 613, 1143

Baumgardt, H., Makino, J., \& Hut, P. 2005, ApJ, 620, 238

Bower, G. C., Wright, M. C. H., Falcke, H., \& Backer, D. C. 2003, ApJ, 588, 331

Feldmeier, A., Lützgendorf, N., Neumayer, N., et al. 2013, A\&A, 554, AA63

Ferrarese, L. \& Merritt, D. 2000, ApJ, 539, L9

Gill, M., Trenti, M., Miller, M. C., et al. 2008, ApJ, 686, 303

Häring, N. \& Rix, H.-W. 2004, ApJ, 604, L89

Hurley, J. R. 2007, MNRAS, 379, 93

Hurley, J. R., Pols, O. R., \& Tout, C. A. 2000, MNRAS, 315, 543

Kruijssen, J. M. D. \& Lützgendorf, N. 2013, MNRAS, 434, L41

Kustaanheimo, P. \& Stiefel, E. 1965, J. Reine Angew. Math., 218, 204

Lanzoni, B., Mucciarelli, A., Origlia, L., et al. 2013, ApJ, 769, 107

Lützgendorf, N., Gebhardt, K., Baumgardt, H., et al. 2015, A\&A, 581, A1

Lützgendorf, N., Baumgardt, H., \& Kruijssen, J. M. D. 2013a, A\&A, 558, A117

Lützgendorf, N., Kissler-Patig, M., Gebhardt, K., et al. 2013b, A\&A, 552, A49

Lützgendorf, N., Kissler-Patig, M., Gebhardt, K., et al. 2012, A\&A, 542, A129

Lützgendorf, N., Kissler-Patig, M., Neumayer, N., et al. 2013c, A\&A, 555, A26

Lützgendorf, N., Kissler-Patig, M., Noyola, E., et al. 2011, A\&A, 533, A36+

Madau, P. \& Rees, M. J. 2001, ApJ, 551, L27

Nayakshin, S. 2005, A\&A, 429, L33

Nitadori, K. \& Aarseth, S. J. 2012, MNRAS, 424, 545

Noyola, E. \& Baumgardt, H. 2011, ApJ, 743, 52

Noyola, E., Gebhardt, K., \& Bergmann, M. 2008, ApJ, 676, 1008

Pasquini, L., Avila, G., Blecha, A., et al. 2002, The Messenger, 110, 1

Pelupessy, F. I., van Elteren, A., de Vries, N., et al. 2013, A\&A, 557, A84

Portegies Zwart, S., McMillan, S., Harfst, S., et al. 2009, New Astronomy, 14, 369

Portegies Zwart, S., McMillan, S. L. W., van Elteren et al. 2013, Comp. Phys. Comm., 183, 456

Portegies Zwart, S. F., Baumgardt, H., Hut, P. et al. 2004, Nature, 428, 724

Strader, J., Chomiuk, L., Maccarone, T. J., et al. 2012, ApJ, 750, L27

Trenti, M., Ardi, E., Mineshige, S., \& Hut, P. 2007, MNRAS, 374, 857

Trenti, M., Vesperini, E., \& Pasquato, M. 2010, ApJ, 708, 1598

Vesperini, E. \& Trenti, M. 2010, ApJ, 720, L179 